\title{
HEART FAILURE PRESENTED WITH MASSIVE PLEURAL EFFUSION AND MEDIASTINAL LYMPHADENOPATHY: A RARE CASE REPORT
}

\author{
MOHAMMAD SHAHIDUL ISLAM ${ }^{1}$, HUMAYARA TABASSUM ${ }^{2}$, SHARAH JAHAN ${ }^{3}$, MOHAMMAD SHAHIN \\ MASUD ${ }^{4}$, MUHAMMAD AL AMIN 5 , AHMEDUL KABIR 6
}

\begin{abstract}
:
Heart failure may present with diverse manifestation. It is far most common cause of bilateral transudative pleural effusion. In very unusual case, it may present with only massive transudative pleural effusion without cardiomegaly and other features of heart failure such as leg odema, tender hepatomegaly or congested liver. We presented a case that present with massive transudative pleural effusion with mediastinal lymphadenopathy evidenced by CT scan of chest and treated successfully with diuretics.
\end{abstract}

Keywords: Chronic Heart Failure; Transudative Pleural Effusion; Mediastinal Lymphadenopathy;

\section{Introduction:}

Heart failure is so common in our country that we face it every day in tertiary hospital. The diagnosis of heart failure is relatively straightforward when the patient presents with classic signs and symptoms of heart failure. Though bilateral pleural effusion is the most common manifestation of heart failure along with other clinical feature, rarely it may present only bilateral pleural effusion. Like that we faced a case in Dhaka Medical College Hospital that presented with bilateral pleural effusion without cardiomegaly 4 months ago.

\section{Case Report:}

This 40-year-old female hailing from sonargaon, a known case of vitiligo since $1997 \&$ diabetes mellitus since 2007 with diabetic proliferative retinopathy admitted to Dhaka Medical College Hospital with fatigue, loss of appetite, nausea, recurrent upper abdominal pain, constipation, shortness of breath occasionally for last five months. She was a two para in 1997. Vitiligo developed following her last child birth at 1997 without any maternal complication, gradually involved the whole body. At 2007, diabetes mellitus was diagnosed and she used insulin from that time. Later she developed diabetic proliferative retinopathy as a consequence of uncontrolled diabetes. For last five months, she felt fatigue, loss of appetite, nausea but had no history of vomiting. As her food intake was not good, she lost her weight significantly. She also felt recurrent upper abdominal pain, colicky in nature, persisted four to five minutes with an interval of one to two minutes. She also mentioned about constipation, bowel moved every four to five days interval. Occasionally she felt shortness of breath. But she did her daily activities at that time. She had no history of nocturnal dyspnoea, photophobia, fever, nocturia and burning micturition. Two days before admission to this hospital, she felt so severe upper abdominal pain that she got admitted in outside of this hospital but after admission, she developed severe shortness of breath, not able to complete a sentence in one breath. According to her husband's statement, her oxygen saturation fell to around $70 \%$. Later she was transferred to ICU. After 1 day, she became stable and transferred to this hospital. On admission, she had anxious looking, moderate anaemia, 160/100 mm $\mathrm{Hg}$ BP, 104 beats/min pulse, jugular venous pressure not raised, no palpable lymphnode and thyroid gland. There was tenderness over epigastric region. Respiratory system revealed reduced chest expansion, reduced vocal fremitus on palpation, dull on percussion, diminished breath sound and reduced vocal resonance on auscultation from $6^{\text {th }}$ intercostals space. There was no abnormality in cardiac examination. Examination of other systems revealed no abnormality. Initial investigation revealed $7.9 \mathrm{gm} /$ dl $\mathrm{Hb} \%, 50 \mathrm{~mm}$ in $1^{\text {st }}$ hour ESR, WBC 8500/cmm.

1. Honorary Medical Officer, Internal Medicine, Dhaka Medical College Hospital

2. Honorary Medical Officer, Internal Medicine, Dhaka Medical College Hospital

3. Honorary Medical Officer, Internal Medicine, Dhaka Medical College Hospital

4. Assistant Registrar, Internal Medicine, Dhaka Medical College Hospital

5. Registrar, Dhaka Internal Medicine, Medical College Hospital

6. Associate Professor, Internal Medicine, Dhaka Medical College Hospital

Bangladesh J Medicine 2013; 24 : 86-88 
Serial troponin I, Serum amylase, serum lipase was within normal range. There was hyponatremia $(\mathrm{Na}+$ $125 \mathrm{mmol} / \mathrm{l})$ with compensatory metabolic acidosis in serum electrolyte report. $\mathrm{X}$ ray chest posterior anterior view revealed bilateral moderate pleural effusion, more on right side than left side. Pleural fluids collected from right side were transudative in nature (protein $1.2 \mathrm{gm} / \mathrm{dl}$ ) and 90\% lymphocytes out of total 70 cells per $\mathrm{cmm}$ present. In ECG, ischemic changes in lead I, II, III, avF, v4- v6. Initial echocardiography exposed mild systolic dysfunction, LVEF- 47\%, anterior wall hypokinesia. Serum creatinine was mildly raised $(1.5 \mathrm{mg} / \mathrm{dl})$. USG of whole abdomen was suggestive of granular calculi or sludge in the gall bladder with mildly thick wall and relatively larger and swollen kidney due to reactionary change. Moderate proteinuria, plenty pus cell was in urine routine microscopic examination. E. coli was identified in urinary culture. Urinary total protein in 24 hours was $0.84 \mathrm{gm}$ in $1200 \mathrm{ml}$. Thyroid function test was normal. Serum total protein was $62.5 \mathrm{gm} /$ litre and serum albumin was $28.0 \mathrm{gm} / 1$. ANA was negative done by indirect immunofluorescent method. All tumour markers were negative. On $7^{\text {th }}$ day of admission, she again developed shortness of breath, left lower chest pain and became drowsy. She became cyanosed, her JVP was raised, 80/50 $\mathrm{mm} \mathrm{Hg}$ and oxygen saturation fall around $77 \%$ although no peripheral oedema, no palpable tender liver. And she was on inotropic support at that time. After 48 hours, she became stable. So again serial troponin I was done and it was negative all the time.

Echocardiography was also done 6 days after $1^{\text {st }}$ echocardiography. It revealed antero-septal-lateralapical hypokinesia, LVEF- 37\%, Moderate LV systolic dysfunction, MR (grade-II), massive pleural effusion with echogenic shadow in left pleural space. CT scan of chest showed bilateral large pleural effusion with collapse- consolidation of adjacent lung field. Inflammatory changes in antero- basal and lateral basal segments of right lower lobes and posterior segment of left lower lobes, mediastinal lymphadenopathy in right para tracheal region. Pleural fluid of left side was also transudative in nature. Serum BNP was very high $(965 \mathrm{pg} / \mathrm{dl})$ done at $20^{\text {th }}$ hospitalization day. We treated this case as Acute on chronic heart failure with diuretics. After 72 hours of starting diuretics, there was no pleural fluid revealed by clinical examination and follow up $\mathrm{X}$ ray chest posterior anterior view. Time to time, patient condition was improved. Follow up CT scan of chest done 2 weeks later revealed no pleural fluid, no lymphadenopathy. One month later, no pus cell in urine routine examination and no growth were identified in urinary culture. In this time, she did not get any antibiotic except linezolid.

\section{Discussion:}

Chronic heart failure commonly follows a relapsing and remitting course, with periods of stabilization and episodes of decompensation leading to worsening symptoms $^{\mathbf{1}}$. That means a patient with chronic heart failure may pass through a phase of asymptomatic LV dysfunction followed by the gradual development of fatigue, exercise tolerance, shortness of breath, leg oedema, ascites and ultimately skeletal muscle wasting or cachexia. Dyspnoea and fatigue are the cardinal symptoms of chronic heart failure. Sometimes patient cannot easily distinguishes between dyspnoea and fatigue and they frequently coexist. In chronic heart failure, adaptive mechanisms are gradually activated, cardiac hypertrophy develops and allows the patient to adjust to and tolerate a reduction in cardiac output with less difficulty. When the onset of left-heart failure is gradual, the right heart develops higher pressures in response to higher pulmonary resistance; the acute onset of similar increases in pulmonary resistance may produce acute right-heart failure $^{2}$. This patient achieved compensation by adaptive mechanism activation. In this case, patient experienced acute decompensation due to uncontrolled hypertension. This hypertension was due to development of diabetic nephropathy evidenced by loss of albumin in urine and renal insufficiency and reactionary large and swollen kidney described by ultra sonography.

The peculiar presentation of this case was patient having raised jugular venous pressure and bilateral large pleural effusion \& mediastinal lymphadenopathy but no ascites, no oedema, no tender congested hepatomagaly. Pleural effusions are a common manifestation of heart failure \& usually associated with other manifestations of heart failure while the dyspnoea is frequently out of proportion to the size of the effusion. Patients who present with the typical findings of cardiomegaly and bilateral pleural effusions probably do not require diagnostic thoracocentesis ${ }^{3}$. However, marked pleural effusion in the absence of cardiomegaly except heart failure due to acute myocardial infarction should raise the suspicion of some other disease and should undergo thoracocentesis as what we did in this case. In one study of 76 patients with bilateral pleural effusions but a normal-sized heart, only $4 \%$ were due to congestive heart failure ${ }^{4}$. For chronic heart failure, pleural effusion are transudates with a low protein concentration (pleural fluid to serum ratio less than 0.5 ) and a low absolute lactate dehydrogenase (LDH) value (less than two-thirds the upper limit of normal) 
and a pleural fluid to serum $\mathrm{LDH}$ ratio of less than $0.6^{\mathbf{5}}$. The fluid typically contains less than 1000 cells / $\mathrm{cmm}$, the majority of which are lymphocytes and mesothelial cells ${ }^{6}$ which completely correlate with our findings. In patients with pleural effusions secondary to chronic heart failure, mediastinal lymphadenopathy is common. One study stated that the prevalence of mediastinal lymphadenopathy with a diameter greater than $1 \mathrm{~cm}$ on the short axis was $81 \%$ with a calculated ejection fraction less than $35 \%{ }^{7}$. We also found para tracheal mediastinal lymphadenopathy in CT scan of chest that misguided us at some point. As pleural effusion was resolved with only diuretic therapy, the diagnosis is considered clinically confirmed. For establishing the diagnosis, serum or pleural fluid BNP should be considered. In clinical practice, levels below $100 \mathrm{pg} / \mathrm{ml}$ are thought to make congestive heart failure unlikely, whereas levels above $500 \mathrm{pg} / \mathrm{ml}$ are considered diagnostic of congestive heart failure ${ }^{\mathbf{8}}$. In this case we found 965 $\mathrm{pg} / \mathrm{ml}$ serum BNP and established our clinical suspicion. In $2^{\text {nd }}$ time echocardiography also revealed an echogenic shadow in left pleural place but in subsequent $\mathrm{CT}$ scan of chest we did not find any mass in that space. We also found E. coli in urinary culture that considered as aymptomatic bacteriuria as she had no fever, burning micturition, renal angle tenderness, and lower abdominal pain.

\section{Conclusion:}

Heart failure is so common in our society that a physician face this situation so many times in clinical practice. But one should rely on clinical intuition to recognition of atypical presentation of heart failure for further testing and treatment though it is very difficult.

\section{References:}

1. Newby DN, Grubb NR, Bradbury A. Cardiovascular Disease : Presenting Problems in Cardiovascular Disease. In Colledge NR, Walker BR, Ralston SH, editors. Davidson's Priniciples \& Practice of Medicine. 21st ed. Edinburgh; 2010. p. 543- 550.

2. Deedwania PC, Carbajal EV. Congestive Heart Failure. In Crawford $\mathrm{MH}$, editor. Current Diagnosis \& Treatment: Cardiology. 3rd ed.: The McGraw-Hill Companies; 2009. p. 203 - 220.

3. Hall WJ, Mayewski R. Diagnostic thoracentesis and pleural biopsy in pleural effusions. Annals of Internal Medicine. 1985; 103(5): p. 799- 802.

4. Rabin CB, Blackman NS. Bilateral pleural effusion: its significance in association with a heart of normal size. Journal of the Mount Sinai Hospital New York. 1957; 24: p. 45- 63.

5. Light RW, Macgregor MI, Luchsinger PC, Ball Jr WC. Pleural effusions: the diagnostic separation of transudates and exudates. Annals of Internal Medicine. 1972; 77: p. 507 - 513.

6. Light RW, Erozan YS, BalL WC. Cells in pleural fluid: their value in differential diagnosis. Archives of Internal Medicine. 1973; 132: p. 854 - 860.

7. Erly WK, Borders RJ, Outwater EK, Zaetta JM, Borders GT. Location, size, and distribution of mediastinal lymph node enlargement in chronic congestive heart failure. Journal of Computer Assisted Tomography. 2003; 27(4): p. 485 - 489.

8. Porcel J, Vives M, Cao G, Esquerda A, Rubio M, Rivas M. Measurement of pro-brain natriuretic peptide in pleural fluid for the diagnosis of pleural effusions due to heart failure. The American Journal of Medicine. 2004; 116(6): p. 417 - 420. 